\title{
Thermal buckling analysis of cross-ply plates based on new displacement field
}

\author{
Widad Ibraheem Majeed \\ Department of Mechanical Engineering, University of Baghdad, Baghdad, 00964, Iraq \\ wedad.ibrahim@coeng.uobaghdad.edu.iq
}

Submitted: 05/08/2019

Revised: $\quad 07 / 01 / 2020$

Accepted: 18/01/2020

\begin{abstract}
A higher-order displacement field is used for the analysis of the thermal buckling of composite plates subjected to thermal load; it is based on a constant " $\mathrm{m}$ ", which is optimized to get results relatively close to those given by 3D elasticity theory. Adequate transverse shear strains distribution through the thickness and free stress surfaces of the plate is satisfied using this theory. Hamilton's principle is used to derive equations of motion, which are solved using Navier-type series for simply supported plates. Thermal buckling of cross-ply laminates with various $\left(\alpha_{2} / \alpha_{1}\right)$ ratios, number of layers, aspect ratios, $\mathrm{E}_{1} / \mathrm{E}_{2}$ ratios, and stacking sequence for thick and thin plates is studied in detail. It is concluded that the obtained results using this displacement field are close to those calculated by 3D elasticity theory and other shear deformation plate theories when $\mathrm{m}=0.05$.
\end{abstract}

Keywords: Critical temperature; Cross plates; Displacement field; Shear deformation theory; Thermal buckling.

\section{INTRODUCTION}

Thermal loads have an important role in several engineering industries, like aerospace and transportation structures, which widely used laminated composite materials; therefore, many researchers have studied thermal buckling of these materials based on different plates theories such as two-dimensional theory of elasticity (2D) (classical, first-order theory and higher-order theory) or three-dimensional theory of elasticity (3D), as provided by Noor et al. (1992).

M. Mansour Mohieddin Ghomshei and Amin Mahmoudi, (2010) implemented method of differential quadrature (DQM) to obtain the critical thermal buckling of the cross-ply thin plate; Le-Chung Shiau et al. (2010) used finite element as a solution method to investigate buckling temperature of angle and cross-ply plates; Mohamed Bourada et al. (2011) developed a new four-variable refined plate theory for thermal buckling analysis of functionally graded material (FGM) sandwich plates; Wael R. Abdul-Majeed et al. (2011) investigated thermal buckling of isotropic thermoelastic thin plates using classical theory and the Rayleigh-Ritz method; Adnan Naji Jameel et al. (2012) investigated buckling of laminates with different boundary conditions subjected to thermal and mechanical loads. They used different plate theories, classical laminated plate theory, higher-order shear deformation plate theory, and Finite element method; Marina Ćetković and Lénárt György, (2016) analyzed critical buckling temperature of angleply laminates using nine-node Lagrangian isoperimetric element to derive finite element model for the problem; M. Cetkovic, (2016) studied buckling temperature of composite plates, analytically by Navier's solution and numerically using the isoperimetric finite element approximation and based on Reddy Layerwise theory and its modified version; Youcef Beldjelili et al. (2016) used a four-variable refined plate theory to analyze hygro-thermo-mechanical bending of sigmoid functionally graded material plate. 
R. Vescovini et al. (2017) used Ritz-based variable-kinematic formulation to study thermal buckling of composite plates and sandwich panels; Ait Atmane et al. (2017) used of an efficient beam theory that takes into account both shear deformation and thickness stretching effects by a parabolic variation for all displacements through the thickness to study bending, buckling, and free vibration of beams made from functionally graded material; Houdayfa Ounis and Mohamed-Ouejdi Belarbi (2017) studied the thermal buckling behavior of laminated plates with rectangular cut-outs using classical plate theory as a base for finite element method; Yufeng Xing and Zekun Wang (2017) concerned the critical buckling temperature of functionally graded thin plates based on classical plate theory; A. Menasria et al. (2017) proposed higher shear deformation theory, in which kinematics use only four variables and variation of transverse shear stress as a trigonometric with free plate surfaces reported, to obtain thermal buckling of plates; F. El-Haina et al. (2017) investigated the thermal buckling of thick plate sandwich functionally graded using sinusoidal shear deformation theory and stress function; Abdelbaki Chikh et al. (2017) presented a new displacement field for higher-order shear deformation theory, containing undetermined integral terms and four unknowns to study thermal buckling of composite plates; Bellifa et al. (2017) used a refined plate theory based on a new displacement field that has undetermined integral variables and contains only four unknowns to study buckling analysis of plates made from functionally graded; H.H. Abdelaziz et al. (2017) developed a simple hyperbolic shear deformation theory that has in-plane displacements field varying hyperbolically through the thickness, to investigate bending, vibration, and buckling of power graded material (PGM) sandwich plate with different boundary conditions.

Fouad Bourada et al. (2018) developed refined plate theory with four variables to analyze buckling of plates made from hybrid functionally graded; in this theory, variation of transverse shear deformation is a parabolic through thickness, satisfying surface conditions of the plate; Behrouz Karami et al. (2018) presented a new sizedependent quasi-3D plate theory, which has parabolic variations of displacements through the thickness, to analyze wave dispersion of functionally graded nanoplates resting on an elastic foundation and in hygrothermal environment; Amina Attia et al. (2018) used an efficient higher-order shear deformation theory contains hyperbolic distributions of the transverse shear strains to analyze thermomechanical bending of temperature-dependent functionally graded plates; A. Younsi et al. (2018) proposed two-dimensional and quasi-three-dimensional higher-order shear deformation theory for bending and free vibration investigation of functionally graded plates based on hyperbolic displacement function; Riad Hamza-Cherif et al. (2018) analyzed Euler-Bernoulli beam model with different boundary conditions to predict the vibration of a single-walled carbon nanotube embedded under thermal effect, using Differential Transform Method; A. Bouhadra et al. (2018) improved a higher shear deformation theory to investigate plates made from functionally graded, by accounting in-plane displacements that have undetermined integral terms and a parabolic variation for vertical displacement through the thickness; M. Benchohra et al. (2018) developed a quasi-3D sinusoidal shear deformation theory accounting for shear deformation and thickness-stretching influences using a trigonometric function for all displacements distribution along thickness, to analyze bending and free vibration for simply supported functionally graded plates; Moussa Abualnour et al. (2018) developed a new quasi-3D shear deformation theory including transverse shear strains parabolic variation to analyze free vibration of the simply supported functionally graded plates; H. Fourn et al. (2018) developed a high-order hyperbolic (HSDT) shear deformation theory based on a new field of displacement, which introduces indeterminate integral variables, which are only four variables, to analyze waves propagation of functionally graduated plates;

Djaloul Zarga et al. (2019) used a simple quasi-3D shear deformation theory based on a displacement field that has five variables and reported new kinematics that contain undetermined integral variables, for thermomechanical bending analysis of functionally graded material sandwich plates; Hadjira Hellal et al. (2019) developed a simple "four-variable shear deformation" plate model, which has a displacement field containing integral terms and transverse shear stress trigonometric variation to study the hygrothermal environment effects on buckling and dynamic of plates rest on elastic foundations; Zoulikha Boukhlif et al. (2019) investigated free vibration of functionally graded (FG) plates resting on elastic foundation using a simple quasi-3D higher shear deformation theory, which considered variation of displacement along thickness and has only four unknowns; Boulefrakh Laid et al. (2019) used quasi-3D hyperbolic shear deformation model for static and dynamic analyses of functionally graded plates resting on foundations with damping effect; Sabrina Boutaleb et al. (2019) studied free vibration analysis of the functionally graded rectangular nanoplates, using theory 
of nonlocal elasticity based on the quasi-3D high shear deformation theory, and a cubic displacement function is used along the thickness; Zaoui et al. (2019) developed a two-dimensional and quasi-three-dimensional shear deformation theories that have a novel displacement field, which includes undetermined integral terms and contains fewer unknowns to model the free vibration of FG plates resting on elastic foundations.

Abdelkader Mahmoudi et al. (2019) developed a refined quasi-three-dimensional shear deformation theory that has a number of unknowns and governing equations, only four fewer than unknown displacement functions taken by other theories, to study thermomechanical response of functionally graded sandwich plates; Lynda Amel Chaabane et al. (2019) used a hyperbolic shear deformation theory to analyze static and dynamic behavior of simply supported FG-beam resting on Winkler-Pasternak foundation types; Fouad Bourada et al. (2019) investigated free vibration analysis of simply supported perfect and imperfect (porous) FG beams using a high-order trigonometric deformation theory that has three unknown and has a parabolic shear deformation variation along the thickness; Mohammed Medani et al. (2019) investigated static and dynamic behavior of plate made from Functionally Graded Carbon Nanotubes, reinforced porous sandwich (PMPV) polymer, and nanocomposite plate, using first-order shear deformation theory; Rafik Meksi et al. (2019) developed a new shear deformation plate theory based on a new displacement field containing integrals, which has four variables, and quasi-parabolic variation of transverse shear stress, to analyze the bending, buckling, and natural frequencies functionally graded material sandwich plates.

In the present work, critical temperature of simply supported laminated composite plate is obtained using high-order shear deformation theory of plate based on displacement field used by Mantari et al. (2011) for laminated plates static and free vibration analyses (they take $\mathrm{m}=0.5$ ), which is optimized in the present work to be $\mathrm{m}=0.05$, which gives critical temperature of laminated plates relatively close to that of the three-dimensional elasticity theory. The effects of many thin and thick plate parameters, such as aspect ratio, $E_{1} / E_{2}$ ratio, $\alpha_{2} / \alpha_{1}$ ratio, and different schemes for cross-ply, are investigated.

\section{DISPLACEMENT FIELD}

The new higher-order theory displacement field used by Mantari et al., 2011, is as follows:

$$
\begin{aligned}
& u(x, y, z)=u_{o}(x, y)-z\left(\frac{\partial w}{\partial x}\right)+f(z) \theta_{1}(x, y) \\
& v(x, y, z)=v_{o}(x, y)-z\left(\frac{\partial w}{\partial y}\right)+f(z) \theta_{2}(x, y) \\
& w(x, y, z)=w_{o}(x, y)
\end{aligned}
$$

where $u_{o}(x, y), v_{o}(x, y), w_{o}(x, y), \theta_{1}(x, y), \theta_{1}(x, y), \theta_{2}(x, y)$ are displacement of plate middle surface, and $f(z)$ represents modified function that give more accurate distribution for shear strain and stress in transverse planes of laminated plate. Assuming free boundary conditions of the plate at its top and bottom surfaces, the displacement field becomes:

$$
\begin{aligned}
& u(x, y, z)=u_{o}(x, y)+z\left(\frac{m \pi}{h} \theta_{1}-\frac{\partial w}{\partial x}\right)+\sin \frac{\pi z}{h} e^{m \cos \left(\frac{\pi z}{h}\right)} \theta_{1} \\
& v(x, y, z)=v_{o}(x, y)+z\left(\frac{m \pi}{h} \theta_{2}-\frac{\partial w}{\partial y}\right)+\sin \frac{\pi z}{h} e^{m \cos \left(\frac{\pi z}{h}\right)} \theta_{2} \\
& w(x, y, z)=w_{0} \\
& \text { where } f(z)=\sin \frac{\pi z}{h} e^{m \cos \left(\frac{\pi z}{h}\right)}+y z, \text { where } y=\frac{\pi m}{h}, \mathrm{~m}=\text { constant }
\end{aligned}
$$

The strain-displacement relations take the form, Reddy (2004). 


$$
\begin{aligned}
& \varepsilon_{\mathrm{xx}}=\frac{\partial \mathrm{u}}{\partial \mathrm{x}}, \varepsilon_{\mathrm{yy}}=\frac{\partial \mathrm{v}}{\partial \mathrm{y}}, \varepsilon_{\mathrm{xz}}=\frac{\partial \mathrm{w}}{\partial \mathrm{z}} \\
& \varepsilon_{x y}=\frac{1}{2}\left(\frac{\partial u}{\partial y}+\frac{\partial \mathrm{v}}{\partial \mathrm{x}}\right)=\frac{1}{2} \gamma_{x y}, \varepsilon_{x z}=\frac{1}{2}\left(\frac{\partial u}{\partial z}+\frac{\partial \mathrm{w}}{\partial \mathrm{x}}\right)=\frac{1}{2} \gamma_{x z} \\
& \varepsilon_{y z}=\frac{1}{2}\left(\frac{\partial v}{\partial z}+\frac{\partial \mathrm{w}}{\partial \mathrm{y}}\right)=\frac{1}{2} \gamma_{y z}
\end{aligned}
$$

Strain as function of displacement field is obtained by substituting Equations $(4,5,6)$ into Equations $(8-13)$ and results in

$$
\begin{aligned}
& \varepsilon_{\mathrm{xx}}=\varepsilon_{\mathrm{xx}}^{0}+\mathrm{z} \varepsilon_{\mathrm{xx}}^{1}+\sin \frac{\pi z}{h} e^{m \cos \left(\frac{\pi z}{h}\right)} \varepsilon_{\mathrm{xx}}^{2} \\
& \varepsilon_{\mathrm{yy}}=\varepsilon_{\mathrm{yy}}^{0}+\mathrm{z} \varepsilon_{\mathrm{yy}}^{1}+\sin \frac{\pi z}{h} e^{m \cos \left(\frac{\pi z}{h}\right)} \varepsilon_{\mathrm{yy}}^{2} \\
& \gamma_{\mathrm{xy}}=\varepsilon_{\mathrm{xy}}^{0}+\mathrm{z} \varepsilon_{\mathrm{xy}}^{1}+\sin \frac{\pi z}{h} e^{m \cos \left(\frac{\pi z}{h}\right)} \varepsilon_{\mathrm{xy}}^{2} \\
& \gamma_{\mathrm{xz}}=\varepsilon_{\mathrm{xz}}^{0}+\left(-m * \sin ^{2}\left(\frac{\pi z}{h}\right)_{+} \cos \frac{\pi z}{h}\right) \frac{\pi}{h} e^{m \cos \left(\frac{\pi z}{h}\right)} \varepsilon_{\mathrm{xz}}^{3} \\
& \gamma_{\mathrm{yz}}=\varepsilon_{\mathrm{yz}}^{0}+\left(-m * \sin ^{2}\left(\frac{\pi z}{h}\right)_{+} \cos \frac{\pi z}{h}\right) \frac{\pi}{h} e^{m \cos \left(\frac{\pi z}{h}\right)} \varepsilon_{\mathrm{yz}}^{3}
\end{aligned}
$$

where

$$
\begin{aligned}
& \left\{\begin{array}{c}
\varepsilon_{x x}^{0} \\
\varepsilon_{y y}^{0} \\
\gamma_{x y}^{0}
\end{array}\right\}=\left\{\begin{array}{c}
\frac{\partial u}{\partial x} \\
\frac{\partial u}{\partial x} \\
\frac{\partial u}{\partial y}+\frac{\partial v}{\partial y}
\end{array}\right\},\left\{\begin{array}{c}
\varepsilon_{x x}^{1} \\
\varepsilon_{y y}^{1} \\
\gamma_{x y}^{1}
\end{array}\right\}=\left\{\begin{array}{c}
\frac{m \pi}{h} \frac{\partial \theta_{1}}{\partial x_{1}}-\frac{\partial^{2} w}{\partial x^{2}} \\
\frac{m \pi}{h} \frac{\partial \theta_{2}}{\partial y}-\frac{\partial^{2} w}{\partial y^{2}} \\
\frac{m \pi}{h} \frac{\partial \theta_{2}}{\partial x_{1}}+\frac{m \pi}{h} \frac{\partial \theta_{2}}{\partial y} \\
-2 \frac{\partial^{2} w}{\partial x \partial y}
\end{array}\right\},\left\{\begin{array}{c}
\varepsilon_{x x}^{2} \\
\varepsilon_{y y}^{2} \\
\gamma_{x y}^{2}
\end{array}\right\}=\left\{\begin{array}{c}
\frac{\partial \theta_{1}}{\partial x} \\
\frac{\partial \theta_{2}}{\partial y} \\
\frac{\partial \theta_{2}}{\partial x}+\frac{\partial \theta_{1}}{\partial y}
\end{array}\right\} \\
& \left\{\begin{array}{c}
\gamma_{x z}^{0} \\
\gamma_{y z}^{0}
\end{array}\right\}=\left\{\begin{array}{l}
m \frac{\pi}{h} \Theta_{1} \\
m \frac{\pi}{h} \Theta_{2}
\end{array}\right\},\left\{\begin{array}{c}
\gamma_{x z}^{3} \\
\gamma_{y z}^{3}
\end{array}\right\}=\left\{\begin{array}{l}
\Theta_{1} \\
\Theta_{2}
\end{array}\right\}
\end{aligned}
$$

\section{ENERGY PRINCIPLES}

Principle of virtual displacements is used to derive equations of motion for thermal buckling of composite plate, Reddy (2004):

$$
0=\int_{0}^{t} \delta U+\delta V
$$

where $\delta U$ is expressed as

$$
\left.\delta \mathrm{U}=\left[\int_{\frac{-h}{2}}^{\frac{h}{2}}\left\{\int_{\Omega}^{k} \sigma_{x x} \delta \varepsilon_{x x}^{k}+\sigma_{y y} \delta \varepsilon_{y y}^{k}+\sigma_{x y} \delta \varepsilon_{x y}^{k}+\sigma_{y z} \delta \varepsilon_{y z}^{k}+\sigma_{x z} \delta \varepsilon_{x z}^{k}\right] \partial x \partial y\right\} \partial z\right]=0
$$




$$
\begin{aligned}
& \delta U=\int\left(N_{1} \delta \varepsilon_{x x}^{0}+M_{1} \delta \varepsilon_{x x}^{1}+P_{1} \delta \varepsilon_{x x}^{2}+N_{2} \delta \varepsilon_{y y}^{0}+M_{2} \delta \varepsilon_{y y}^{1}+P_{2} \delta \varepsilon_{y y}^{2}+N_{6} \delta \varepsilon_{X y}^{0}+\right. \\
& \left.M_{6} \delta \varepsilon_{X y}^{1}+P_{6} \delta \varepsilon_{x y}^{2}+Q_{2} \delta \varepsilon_{y z}^{0}+k_{2} \delta \varepsilon_{y z}^{3}+Q_{1} \delta \varepsilon_{\mathrm{xz}}^{0}+k_{1} \delta \varepsilon_{\mathrm{xz}}^{3}-\right) \partial x \partial y=0
\end{aligned}
$$

where $\mathrm{N}_{\mathrm{i}}, \mathrm{Mi}, \mathrm{Pi}$, Qi and $\mathrm{K}_{\mathrm{i}}$ result from the following integration:

$\left(\mathrm{N}_{\mathrm{i}}, \mathrm{M}_{\mathrm{i}}, \mathrm{P}_{\mathrm{i}}\right)=\sum_{k=1}^{N} \int_{z^{k-1}}^{z^{k}} \sigma_{i}^{k}\left(1, z, \sin \frac{\pi z}{h} e^{m \cos \left(\frac{\pi z}{h}\right)}\right) d z \quad(i=1,2,6)$

$\left(\mathrm{Q}_{1}, \mathrm{~K} 1\right)=\sum_{k=1}^{N} \int_{z^{k-1}}^{z^{k}} \sigma_{5}^{k}\left(1, \frac{\pi}{h}\left(-m * \sin ^{2}\left(\frac{\pi z}{h}\right)+\cos \frac{\pi z}{h}\right) e^{m \cos \left(\frac{\pi z}{h}\right)}\right) d z$

$\left(\mathrm{Q}_{2}, \mathrm{~K} 2\right)=\sum_{k=1}^{N} \int_{z^{k-1}}^{z^{k}} \sigma_{4}^{k}\left(1, \frac{\pi}{h}\left(-m * \sin ^{2}\left(\frac{\pi z}{h}\right)+\cos \frac{\pi z}{h}\right) e^{m \cos \left(\frac{\pi z}{h}\right)}\right) d z$

substituting strains from Equations (14-23) into Equation (26) and integrating by parts, then we get

$0=-\int\left[\frac{\partial \mathrm{N}_{1}}{\partial \mathrm{x}} \delta \mathrm{u}+\frac{\mathrm{m} \pi}{\mathrm{h}} \frac{\partial \mathrm{M}_{1}}{\partial \mathrm{x}} \delta \Theta_{1}-\frac{\partial^{2} \mathrm{M}_{1}}{\partial \mathrm{x}^{2}} \delta \mathrm{w}+\frac{\partial \mathrm{P}_{1}}{\partial \mathrm{x}} \delta \Theta_{1}+\frac{\partial \mathrm{N}_{2}}{\partial \mathrm{y}} \delta \mathrm{v}+\frac{\mathrm{m} \pi}{\mathrm{h}} \frac{\partial \mathrm{M}_{2}}{\partial \mathrm{y}} \delta \Theta_{2}-\frac{\partial^{2} \mathrm{M}_{2}}{\partial \mathrm{y}^{2}} \delta \mathrm{w}+\right.$

$\frac{\partial \mathrm{P}_{2}}{\partial \mathrm{y}} \delta \Theta_{2}+\frac{\partial \mathrm{N}_{6}}{\partial \mathrm{y}} \delta \mathrm{u}+\frac{\partial \mathrm{N}_{6}}{\partial \mathrm{x}} \delta \mathrm{v}+\frac{\mathrm{m} \pi}{\mathrm{h}} \frac{\partial \mathrm{M}_{6}}{\partial \mathrm{y}} \delta \Theta_{1}+\frac{\mathrm{m} \pi}{\mathrm{h}} \frac{\partial \mathrm{M}_{6}}{\partial \mathrm{x}} \delta \Theta_{2}+2 \frac{\partial^{2} \mathrm{M}_{6}}{\partial \mathrm{x} \partial \mathrm{y}} \delta \mathrm{w}+\frac{\partial \mathrm{P}_{6}}{\partial \mathrm{y}} \delta \Theta_{1}+\frac{\partial \mathrm{P}_{6}}{\partial \mathrm{x}} \delta \Theta_{2}-$

$\left.\frac{\mathrm{m} \pi}{\mathrm{h}} \mathrm{Q}_{1} \delta \Theta_{1}-\frac{\mathrm{m} \pi}{\mathrm{h}} \mathrm{Q}_{2}-\mathrm{K}_{1} \delta \Theta_{1}-\mathrm{K}_{2} \delta \Theta_{2}\right] \partial \mathrm{x} \partial \mathrm{y}$

And virtual work done by thermal load $\delta V$ is

$$
\delta V=\int_{\Omega}\left\{N_{x}^{T} \delta\left(\frac{\partial w}{\partial x}\right)^{2}+N_{y}^{T} \delta\left(\frac{\partial w}{\partial y}\right)^{2}+N_{x y}^{T} \delta\left(\frac{\partial w}{\partial x}\right) \times\left(\frac{\partial w}{\partial y}\right)\right\} d x d y
$$

\section{EQUATIONS OF MOTION}

Substituting Equations (27-28) into Equation (24), also equating coefficients of ( $\delta \mathrm{u}, \delta \mathrm{v}, \delta \mathrm{w}, \delta \Theta_{1} \delta \Theta_{2}$ ) over $\Omega_{0}$ of Equation (24) to zero separately will result the following equations of motion:

$$
\begin{aligned}
& \delta \mathrm{u}: \frac{\partial \mathrm{N}_{\mathrm{x}}}{\partial \mathrm{x}}+\frac{\partial \mathrm{N}_{\mathrm{xy}}}{\partial \mathrm{y}}=0 \\
& \delta \mathrm{v}: \frac{\partial \mathrm{N}_{\mathrm{y}}}{\partial \mathrm{y}}+\frac{\partial \mathrm{N}_{\mathrm{xy}}}{\partial \mathrm{x}}=0 \\
& \delta \mathrm{w}: \frac{\partial^{2} \mathrm{M}_{\mathrm{x}}}{\partial \mathrm{x}^{2}}+\frac{\partial^{2} \mathrm{M}_{\mathrm{y}}}{\partial \mathrm{y}^{2}}+2 \frac{\partial^{2} \mathrm{M}_{\mathrm{xy}}}{\partial \mathrm{x} \partial \mathrm{y}}+N_{x}^{T}\left(\frac{\partial^{2} w}{\partial x^{2}}\right)+N_{y}^{T}\left(\frac{\partial^{2} w}{\partial y^{2}}\right)=0 \\
& \delta \Theta_{1}: \frac{\mathrm{m} \pi}{\mathrm{h}} \frac{\partial \mathrm{M}_{\mathrm{x}}}{\partial \mathrm{x}}+\frac{\mathrm{m} \pi}{\mathrm{h}} \frac{\partial \mathrm{M}_{\mathrm{xy}}}{\partial \mathrm{y}}+\frac{\partial \mathrm{P}_{\mathrm{x}}}{\partial \mathrm{x}}+\frac{\partial \mathrm{P}_{\mathrm{xy}}}{\partial \mathrm{y}}-\frac{\mathrm{m} \pi}{\mathrm{h}} \mathrm{Q}_{\mathrm{x}}-\mathrm{K}_{\mathrm{x}}=0 \\
& \delta \Theta_{2}: \frac{\mathrm{m} \pi}{\mathrm{h}} \frac{\partial \mathrm{M}_{\mathrm{y}}}{\partial \mathrm{y}}+\frac{\mathrm{m} \pi}{\mathrm{h}} \frac{\partial \mathrm{M}_{\mathrm{xy}}}{\partial \mathrm{x}}+\frac{\partial \mathrm{P}_{\mathrm{y}}}{\partial \mathrm{y}}+\frac{\partial \mathrm{P}_{\mathrm{xy}}}{\partial \mathrm{x}}-\frac{\mathrm{m} \pi}{\mathrm{h}} \mathrm{Q}_{\mathrm{y}}-\mathrm{K}_{\mathrm{y}}=0
\end{aligned}
$$

The two-dimensional plate reduced stiffness $Q_{i j}$ is $Q_{11}=\frac{E_{1}}{1-v_{12} v_{21}}, Q_{12}=\frac{v_{12} E_{2}}{1-v_{12} v_{21}}, Q_{11}=\frac{E_{2}}{1-v_{12} v_{21}} Q_{66}=G_{12}$, $\mathrm{Q}_{44}=\mathrm{G}_{23}, \mathrm{Q}_{55}=\mathrm{G}_{13}$

where $E_{1}$ and $E_{2}=$ Young's modulus in directions 1 and 2 of composite plate, while $G_{12}, G_{23}$ and $G_{13}=$ shear modulus of plate in three orthogonal planes. Also, $v_{12}$ and $v_{21}$ are poison's ratio in plane 1-2. Orthotropic lamina stress 
components are related to strain as follows:

$$
\left\{\begin{array}{c}
\sigma_{\mathrm{xx}} \\
\sigma_{\mathrm{yy}} \\
\sigma_{\mathrm{xy}}
\end{array}\right\}=\left[\begin{array}{l}
\mathrm{Q}_{11} \mathrm{Q}_{12} \mathrm{Q}_{16} \\
\mathrm{Q}_{12} \mathrm{Q}_{22} \mathrm{Q}_{26} \\
\mathrm{Q}_{16} \mathrm{Q}_{26} \mathrm{Q}_{66}
\end{array}\right]\left\{\begin{array}{c}
\varepsilon_{\mathrm{xx}}-\alpha_{\mathrm{xx}} \Delta \mathrm{T} \\
\varepsilon_{\mathrm{yy}}-\alpha_{\mathrm{xx}} \Delta \mathrm{T} \\
\gamma_{\mathrm{xy}-} 2 \alpha_{\mathrm{xy}} \Delta \mathrm{T}
\end{array}\right\},\left\{\begin{array}{l}
\sigma_{\mathrm{yz}} \\
\sigma_{\mathrm{xz}}
\end{array}\right\}=\left[\begin{array}{ll}
\mathrm{Q}_{44} & \mathrm{Q}_{45} \\
\mathrm{Q}_{45} & \mathrm{Q}_{55}
\end{array}\right]\left\{\begin{array}{c}
\gamma_{\mathrm{yz}} \\
\gamma_{\mathrm{xz}}
\end{array}\right\}
$$

Also resulting forces are related to the strains as follows:

$$
\left\{\begin{array}{c}
\mathrm{N}_{\mathrm{x}} \\
\mathrm{N}_{\mathrm{y}} \\
\mathrm{N}_{\mathrm{xy}} \\
\mathrm{M}_{\mathrm{x}} \\
\mathrm{M}_{\mathrm{y}} \\
\mathrm{M}_{\mathrm{xy}} \\
\mathrm{P}_{\mathrm{x}} \\
\mathrm{P}_{\mathrm{y}} \\
\mathrm{P}_{\mathrm{xy}}
\end{array}\right\}=\left[\begin{array}{lllll}
\mathrm{A}_{11} \mathrm{~A}_{12} \mathrm{~A}_{16} & \mathrm{~B}_{11} \mathrm{~B}_{12} \mathrm{~B}_{16} \mathrm{E}_{11} \mathrm{E}_{12} \mathrm{E}_{16} \\
\mathrm{~A}_{12} \mathrm{~A}_{22} \mathrm{~A}_{26} & \mathrm{~B}_{12} \mathrm{~B}_{22} \mathrm{~B}_{26} \mathrm{E}_{12} \mathrm{E}_{22} \mathrm{E}_{26} \\
\mathrm{~A}_{16} \mathrm{~A}_{26} \mathrm{~A}_{66} & \mathrm{~B}_{16} \mathrm{~B}_{26} \mathrm{~B}_{66} & \mathrm{E}_{16} \mathrm{E}_{26} \mathrm{E}_{66} \\
\mathrm{~B}_{11} \mathrm{~B}_{12} \mathrm{~B}_{16} & \mathrm{D}_{11} \mathrm{D}_{12} \mathrm{D}_{16} & \mathrm{~F}_{11} \mathrm{~F}_{12} \mathrm{~F}_{16} \\
\mathrm{~B}_{12} \mathrm{~B}_{22} \mathrm{~B}_{26} & \mathrm{D}_{12} \mathrm{D}_{22} \mathrm{D}_{26} & \mathrm{~F}_{12} \mathrm{~F}_{22} \mathrm{~F}_{26} \\
\mathrm{~B}_{16} \mathrm{~B}_{26} \mathrm{~B}_{66} & \mathrm{D}_{16} \mathrm{D}_{26} \mathrm{D}_{66} & \mathrm{~F}_{16} \mathrm{~F}_{26} \mathrm{~F}_{66} \\
\mathrm{E}_{11} \mathrm{E}_{12} \mathrm{E}_{16} & \mathrm{~F}_{11} \mathrm{~F}_{12} \mathrm{~F}_{16} \mathrm{H}_{11} \mathrm{H}_{12} \mathrm{H}_{16} \\
\mathrm{E}_{12} \mathrm{E}_{22} \mathrm{E}_{26} & \mathrm{~F}_{12} \mathrm{~F}_{22} \mathrm{~F}_{26} & \mathrm{H}_{12} \mathrm{H}_{22} \mathrm{H}_{26} \\
\mathrm{E}_{16} \mathrm{E}_{26} \mathrm{E}_{66} & \mathrm{~F}_{16} \mathrm{~F}_{26} \mathrm{~F}_{66} & \mathrm{H}_{16} \mathrm{H}_{26} \mathrm{H}_{66}
\end{array}\right]\left\{\begin{array}{c}
\varepsilon_{\mathrm{y}}^{0} \\
\varepsilon_{\mathrm{xy}}^{0} \\
\varepsilon_{\mathrm{x}}^{1} \\
\varepsilon_{\mathrm{y}}^{1} \\
\varepsilon_{\mathrm{xy}}^{1} \\
\varepsilon_{\mathrm{x}}^{2} \\
\varepsilon_{\mathrm{y}}^{2} \\
\varepsilon_{\mathrm{xy}}^{2}
\end{array}\right\}
$$

$$
\left\{\begin{array}{l}
\mathrm{Q}_{\mathrm{x}} \\
\mathrm{Q}_{\mathrm{y}} \\
\mathrm{K}_{\mathrm{x}} \\
\mathrm{K}_{\mathrm{y}}
\end{array}\right\}=\left[\begin{array}{cccc}
\mathrm{A}_{44} & \mathrm{~A}_{45} \mathrm{~J}_{44} & \mathrm{~J}_{45} \\
\mathrm{~A}_{45} & \mathrm{~A}_{55} \mathrm{~J}_{45} & \mathrm{~J}_{55} \\
\mathrm{~J}_{44} & \mathrm{~J}_{45} \mathrm{~L}_{44} & \mathrm{~L}_{45} \\
\mathrm{~J}_{45} & \mathrm{~J}_{55} \mathrm{~L}_{45} & \mathrm{~L}_{55}
\end{array}\right]\left\{\begin{array}{c}
\gamma_{\mathrm{yz}}^{0} \\
\gamma_{\mathrm{xz}}^{0} \\
\gamma_{\mathrm{yz}}^{3} \\
\gamma_{\mathrm{xz}}^{3}
\end{array}\right\}
$$

while in plane thermal loads are

$$
\left\{\begin{array}{c}
N_{x}^{T} \\
N_{y}^{T} \\
N_{x y}^{T}
\end{array}\right\}=\sum_{k=1}^{N} \int_{z^{k}}^{z^{k+1}}\left[\begin{array}{lll}
\mathrm{Q}_{11} & \mathrm{Q}_{12} & \mathrm{Q}_{16} \\
\mathrm{Q}_{12} & \mathrm{Q}_{22} & \mathrm{Q}_{26} \\
\mathrm{Q}_{16} & \mathrm{Q}_{26} & \mathrm{Q}_{66}
\end{array}\right]\left\{\begin{array}{c}
\alpha_{x x} \\
\alpha_{y y} \\
2 \alpha_{x y}
\end{array}\right\} \Delta \mathrm{T} d z
$$

where $A_{i j}=\int_{\frac{-h}{2}}^{\frac{h}{2}} Q_{i j} d z \quad i=(1,2,4,5,6)$

$$
\left(\mathrm{B}_{\mathrm{ij}}, \mathrm{D}_{\mathrm{ij}}, \mathrm{E}_{\mathrm{ij}}\right)=\int_{\frac{-\mathrm{h}}{2}}^{\frac{\mathrm{h}}{2}} \mathrm{Q}_{\mathrm{ij}}\left(\mathrm{z}, \mathrm{z}^{2}, \sin \left(\frac{\pi \mathrm{z}}{\mathrm{h}}\right) e^{m \cos \left(\frac{\pi z}{h}\right)}, \quad(\mathrm{i}, \mathrm{j}=1,2,6)\right.
$$

$$
\left(\mathrm{F}_{\mathrm{ij}}, \mathrm{H}_{\mathrm{ij}}\right)=\int_{\frac{-\mathrm{h}}{2}}^{\frac{\mathrm{h}}{2}} \mathrm{Q}_{\mathrm{ij}}\left(\sin \left(\frac{\pi \mathrm{z}}{\mathrm{h}}\right) e^{m \cos \left(\frac{\pi \mathrm{z}}{h}\right)} * \mathrm{z}, \sin ^{2}\left(\frac{\pi \mathrm{z}}{\mathrm{h}}\right) e^{2 m \cos \left(\frac{\pi \mathrm{z}}{h}\right)}\right) \quad(\mathrm{i}, \mathrm{j}=1,2,6)
$$

$\mathrm{J}_{\mathrm{ij}}=\int_{\frac{-\mathrm{h}}{2}}^{\frac{\mathrm{h}}{2}} \mathrm{Q}_{\mathrm{ij}} \frac{\pi}{\mathrm{h}} e^{m \cos \left(\frac{\pi \mathrm{z}}{h}\right)}\left(-\mathrm{m} * \sin ^{2}\left(\frac{\pi \mathrm{z}}{\mathrm{h}}\right)+\cos \frac{\pi \mathrm{z}}{\mathrm{h}}\right) \mathrm{dz}$

$$
\mathrm{L}_{\mathrm{ij}}=\int_{\frac{-\mathrm{h}}{2}}^{\frac{\mathrm{h}}{2}} \mathrm{Q}_{\mathrm{ij}}\left(\frac{\pi}{\mathrm{h}}\right)^{2} e^{2 m \cos \left(\frac{\pi z}{h}\right)}\left(-\mathrm{m} * \sin ^{2}\left(\frac{\pi \mathrm{z}}{\mathrm{h}}\right)+\cos \frac{\pi \mathrm{z}}{\mathrm{h}}\right)^{2} \mathrm{dz} \quad \mathrm{i}, \mathrm{j}=(4,5)
$$

And, $\alpha_{x x}, \alpha_{y y}, \alpha_{x y}$ are coefficients of thermal expansion for composite plate. 


\section{NAVIER'S SOLUTION}

Simply supported boundary conditions for cross-ply plate are satisfied by Navier's generalized displacements, which are trigonometric series in terms of unknown amplitudes as shown below Reddy (2004):

$$
\begin{aligned}
& \mathrm{u}(\mathrm{x}, \mathrm{y}, \mathrm{t})=\sum_{\mathrm{M}=1}^{\infty} \sum_{\mathrm{N}=1}^{\infty} \mathrm{U}_{\mathrm{MN}} \cos (\alpha \mathrm{x}) \sin (\beta \mathrm{y}) \\
& \mathrm{v}(\mathrm{x}, \mathrm{y}, \mathrm{t})=\sum_{\mathrm{M}=1}^{\infty} \sum_{\mathrm{N}=1}^{\infty} \mathrm{V}_{\mathrm{MN}} \sin (\alpha \mathrm{x}) \cos (\beta \mathrm{y}) \\
& \mathrm{w}(\mathrm{x}, \mathrm{y}, \mathrm{t})=\sum_{\mathrm{M}=1}^{\infty} \sum_{\mathrm{N}=1}^{\infty} \mathrm{W}_{\mathrm{MN}} \sin (\alpha \mathrm{x}) \sin (\beta \mathrm{y}) \\
& \theta_{1}(\mathrm{x}, \mathrm{y}, \mathrm{t})=\sum_{\mathrm{M}=1}^{\infty} \sum_{\mathrm{N}=1}^{\infty} \theta_{1 \mathrm{MN}} \cos (\alpha \mathrm{x}) \sin (\beta \mathrm{y}) \\
& \theta_{2}(\mathrm{x}, \mathrm{y}, \mathrm{t})=\sum_{\mathrm{M}=1}^{\infty} \sum_{\mathrm{N}=1}^{\infty} \theta_{2 \mathrm{MN}} \sin (\alpha \mathrm{x}) \cos (\beta \mathrm{y})
\end{aligned}
$$

And: $\alpha=\frac{M \pi}{h}, \beta=\frac{N \pi}{h},\left(U_{M N}, V_{M N}, W_{M N}, \theta_{1 \mathrm{MN}}, \theta_{2 \mathrm{MN}}\right)$, are unknown amplitudes.

\section{EIGNVALUE PROBLEM}

Express equations of motion (29-33) in terms of displacements by using Equations (36 - 43) and then solved using Equations (44-48), and the following eigenvalue problem is developed:

$$
\left(\left[\begin{array}{ccccc}
c_{11} & c_{12} & c_{13} & c_{14} & c_{15} \\
& c_{22} & c_{23} & c_{24} & c_{25} \\
& & c_{33-\alpha^{2} N_{x}^{T}-\beta^{2} N_{y}^{T}-\alpha \beta N_{x y}^{T}} & c_{34} & c_{35} \\
& & & c_{44} & c_{45} \\
& & & & c_{55}
\end{array}\right)\{d\}=0\right.
$$

where $\left\{\mathrm{d}_{\mathrm{ij}}\right\}=\left\{\mathrm{U}_{\mathrm{mn}}, \mathrm{V}_{\mathrm{mn}}, \mathrm{W}_{\mathrm{mn}}, \theta_{1_{\mathrm{mn}}}, \theta_{2 \mathrm{mn}}\right\}$

And $C_{i j}$ is the element of stiffness, from its determinant thermal buckling is obtained.

\section{RESULTS AND DISCUSSION}

Using the above analytical solutions of the HOSDT based on displacement function given by J.L. Mantari et al. (2011), a computer program is built using MATLAB (R2015a). Design parameters effect such as thickness ration (a/h), aspect ratio $(a / b)$, orthotropy ratio $E_{1} / E_{2}$ and coefficient of expansion ratio $\left(\alpha_{2} / \alpha_{1}\right)$ on thermal buckling of orthotropic plates are investigated. Suggested above solution is verified by comparing present results with those given by other researchers and give good agreement as shown in Table (1), for different number of symmetric cross-ply and different thickness ratio $(\mathrm{a} / \mathrm{h})$, and for antisymmetric cross-ply plate and with 3-dimensional solution proposed by Noor et al., 1992 as listed in Table (2). 
Table 1. Normalized critical temperature $\left(T^{*} \mathrm{cr}=\mathrm{T}^{*} \mathrm{a}^{2 *} \mathrm{~h} / \pi^{2 *} \mathrm{D}_{22}\right)$ for symmetric cross-ply square plate, mode $(\mathrm{M}=1, \mathrm{~N}=1)$.

\begin{tabular}{|c|c|c|c|c|}
\hline \multirow{3}{*}{$\mathbf{a} / \mathbf{h}$} & \multirow{2}{*}{ References } & \multicolumn{3}{c|}{ Tcr } \\
\cline { 2 - 5 } & Present & 4layers & 8layers & 20layers \\
\hline \multirow{3}{*}{4} & L. X. SUN and T. R. HSIJ, 1990 & 0.0533 & 0.03113 & .02478 \\
\cline { 2 - 5 } & Shu Xiaopingt and Sun Liangxint, 1994 & 0.0554 & 0.0315 & 0.0247 \\
\cline { 2 - 5 } & Present & 0.14199 & 0.0279 & 0.0218 \\
\cline { 2 - 5 } 10 & L. X. SUN and T. R. HSIJ, 1990 & 0.1522 & 0.07737 & 0.06094 \\
\cline { 2 - 5 } & Shu Xiaopingt and Sun Liangxint, 1994 & 0.1436 & 0.0747 & .0621 \\
\hline \multirow{3}{*}{100} & Present & 0.2364 & 0.11255 & 0.0584 \\
\cline { 2 - 5 } & L. X. SUN and T. R. HSIJ, 1990 & 0.2435 & 0.1148 & 0.0856 \\
\cline { 2 - 5 } & Shu Xiaopingt and Sun Liangxint, 1994 & 0.2431 & 0.1147 & 0.0872 \\
\hline
\end{tabular}

Table 2. Normalized critical temperature $\left(\mathrm{Tcr}=\mathrm{T}^{*} 1 \mathrm{e}-6\right)$ for cross-ply plate $(\mathrm{a} / \mathrm{b}=1), \mathrm{E}_{1} / \mathrm{E}_{2}=15, \mathrm{G}_{12}=\mathrm{G}_{13}=.5 \mathrm{E}_{2}$, $\mathrm{G}_{23}=.3356 \mathrm{E}_{2}, v_{12}=0.3, \alpha_{2} / \alpha_{1}=0.015$.

\begin{tabular}{|c|c|c|c|c|c|}
\hline \multirow[t]{2}{*}{$\mathbf{a} / \mathbf{h}$} & \multicolumn{2}{|c|}{$\begin{array}{c}(\mathbf{0} / 90) \\
\operatorname{mode}(M=1, N=1)\end{array}$} & \multicolumn{2}{|c|}{$\begin{array}{c}{[0]} \\
\operatorname{mode}(\mathbf{M}=1, N=2)\end{array}$} & \multirow{2}{*}{$\begin{array}{l}\text { Discrepancy \% } \\
\text { (present and Noor } \\
\text { et al. (1992)) }\end{array}$} \\
\hline & References & Ter & References & Ter & \\
\hline \multirow{2}{*}{4} & Present & 0.1972 & Present & 0.18717 & \multirow{2}{*}{5.05} \\
\hline & M. Cetkovic, 2016 & 0.1834 & Noor et al. 1992 & 0.1777 & \\
\hline \multirow{2}{*}{10} & Present & 0.0457 & Present & 0.05923 & \multirow{2}{*}{2.3} \\
\hline & M. Cetkovic, 2016 & $0.4397 * 10^{-1}$ & Noor et al. 1992 & $0.5782 \times 10^{-1}$ & \\
\hline \multirow{2}{*}{20} & Present & 0.0122 & Present & 0.01755 & \multirow{2}{*}{0.911} \\
\hline & M. Cetkovic, 2016 & $0.1184 * 10^{-1}$ & Noor et al. 1992 & $0.1739 \times 10^{-1}$ & \\
\hline \multirow{2}{*}{100} & Present & 0.0005007 & Present & 0.0007479 & \multirow{2}{*}{0.213} \\
\hline & M. Cetkovic, 2016 & $0.4857 * 10^{-3}$ & Noor et al. 1992 & $0.7463 \times 10^{-3}$ & \\
\hline
\end{tabular}

Changing thermal expansion coefficient ratio $\left(\alpha_{2} / \alpha_{1}\right)$ effect on critical buckling temperature of 8layer thick and thin plates (with different (a/h) ratio), are listed in Table (3) and shown in Fig.(1), as expected critical temperature decrease when $\left(\alpha_{2} / \alpha_{1}\right)$ increase and $(\mathrm{a} / \mathrm{H})$ increase since stiffness decrease when plate become thinner. 
Table 3. Effect of $\left(\alpha_{2} / \alpha_{1}\right)$ on normalized critical temperature $\left(T^{*} \mathrm{cr}=\mathrm{T}^{*} \mathrm{a}^{2 *} \mathrm{~h} / \pi^{2 *} \mathrm{D}_{22}\right)$ for symmetric cross-ply square plate, mode $(\mathrm{M}=1, \mathrm{~N}=1)$, No. of layers $=8$.

\begin{tabular}{|c|c|c|}
\hline $\mathbf{a} / \mathbf{h}$ & $\left(\alpha_{2} / \alpha_{1}\right)$ & $\mathbf{T}^{*} \mathrm{cr}$ \\
\hline \multirow{5}{*}{4} & 2 & 0.03268 \\
\hline & 4 & 0.02973 \\
\hline & 6 & 0.02726 \\
\hline & 8 & 0.02517 \\
\hline & 10 & 0.02338 \\
\hline \multirow{5}{*}{10} & 2 & 0.08174 \\
\hline & 4 & 0.07435 \\
\hline & 6 & 0.06819 \\
\hline & 8 & 0.06297 \\
\hline & 10 & 0.05849 \\
\hline \multirow{5}{*}{100} & 2 & 0.11814 \\
\hline & 4 & 0.10746 \\
\hline & 6 & 0.09856 \\
\hline & 8 & 0.09101 \\
\hline & 10 & 0.08454 \\
\hline
\end{tabular}

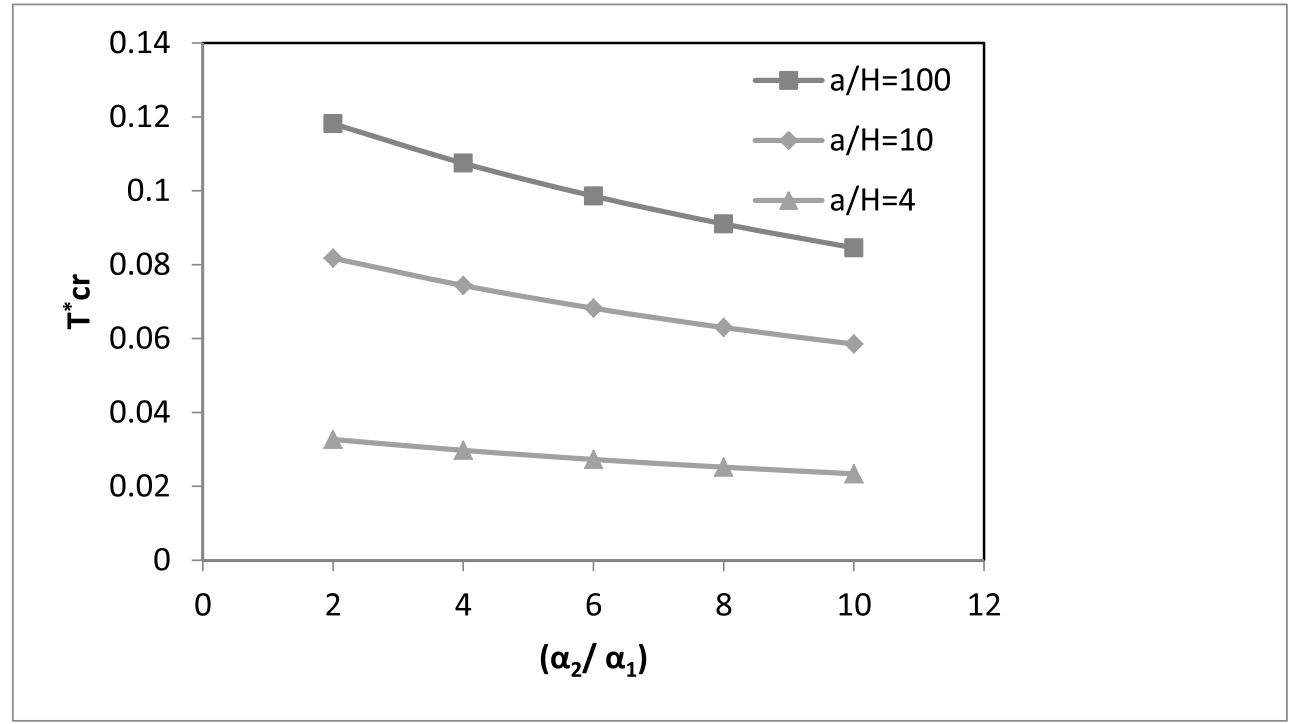

Figure 1. Effect of $\left(\alpha_{2} / \alpha_{1}\right)$ on normalized critical temperature $\left(T^{*} \mathrm{cr}=\mathrm{T}^{*} \mathrm{a}^{2 *} \mathrm{~h} / \pi^{2 *} \mathrm{D}_{22}\right)$ for symmetric cross-ply square plate, mode $(\mathrm{M}=2, \mathrm{~N}=1)$, No. of layers $=8$. 
Aspect ratio effect on critical thermal buckling of 4layer thick and thin composite plates, are listed in Table (4), normalized critical temperature increase as aspect ratio increase because decreasing stiffness $\left(D_{22}\right)$ or critical temperature decrease as $(\mathrm{a} / \mathrm{b})$ increase, also critical temperature for antisymmetric cross-ply is larger than that for symmetric since stiffness is better for the former. Different critical thermal buckling modes for plates with different aspect ratio are shown in Figs. (2-5).

Table 4. Effect of $(\mathrm{a} / \mathrm{b})$ on normalized critical temperature $\left(T^{*} \mathrm{cr}=\mathrm{T}^{*} \mathrm{a}^{2 *} \mathrm{~h} / \pi^{2 *} \mathrm{D}_{22}\right)$ for cross-ply square plate, mode $(\mathrm{M}=1, \mathrm{~N}=1)$, No. of layers $=4$.

\begin{tabular}{|c|c|c|c|c|c|c|}
\hline \multirow{2}{*}{$\mathbf{a} / \mathbf{b}$} & \multicolumn{3}{|c|}{ Symmetric layers } & \multicolumn{3}{c|}{ Antisymmetric layers } \\
\cline { 2 - 7 } & \multicolumn{3}{|c|}{$\mathbf{a} / \mathbf{h} / \mathbf{c}$} \\
\cline { 2 - 7 } & 4 & 10 & 100 & 4 & 10 & 100 \\
\hline 1 & 0.05338 & 0.14199 & 0.23647 & 0.01974 & 0.04642 & 0.06298 \\
\hline 2 & 0.06925 & 0.19843 & 0.32143 & 0.02613 & 0.09148 & 0.20054 \\
\hline 3 & 0.08183 & 0.29227 & 0.61414 & 0.03170 & 0.12690 & $\begin{array}{c}0.44651 \\
(\mathrm{M}=2, \mathrm{~N}=1)\end{array}$ \\
\hline 4 & 0.09136 & 0.36841 & 1.05542 & $\begin{array}{c}0.03714 \\
(\mathrm{M}=2, \mathrm{~N}=1)\end{array}$ & $\begin{array}{c}0.14713 \\
(\mathrm{M}=2, \mathrm{~N}=1)\end{array}$ & $\begin{array}{c}0.77310 \\
(\mathrm{M}=2, \mathrm{~N}=1)\end{array}$ \\
\hline
\end{tabular}

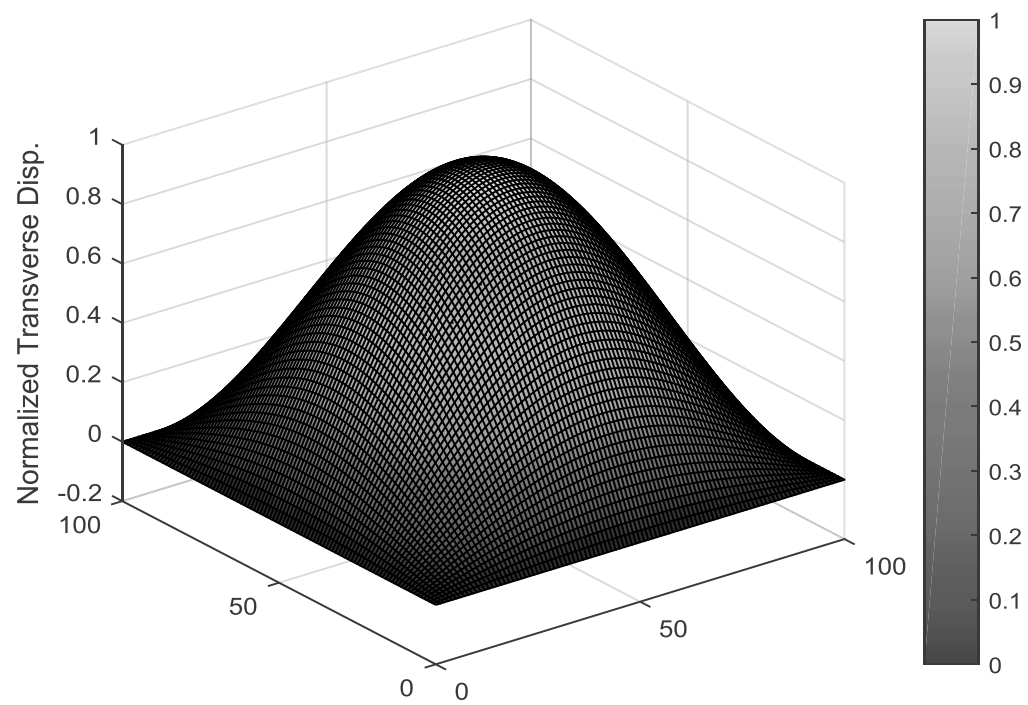

Figure 2. Thermal Buckling mode for symmetric cross-ply square plate, mode $(M=1, N=1)$, No. of layers $=8, a / h=4, a / b=1$. 


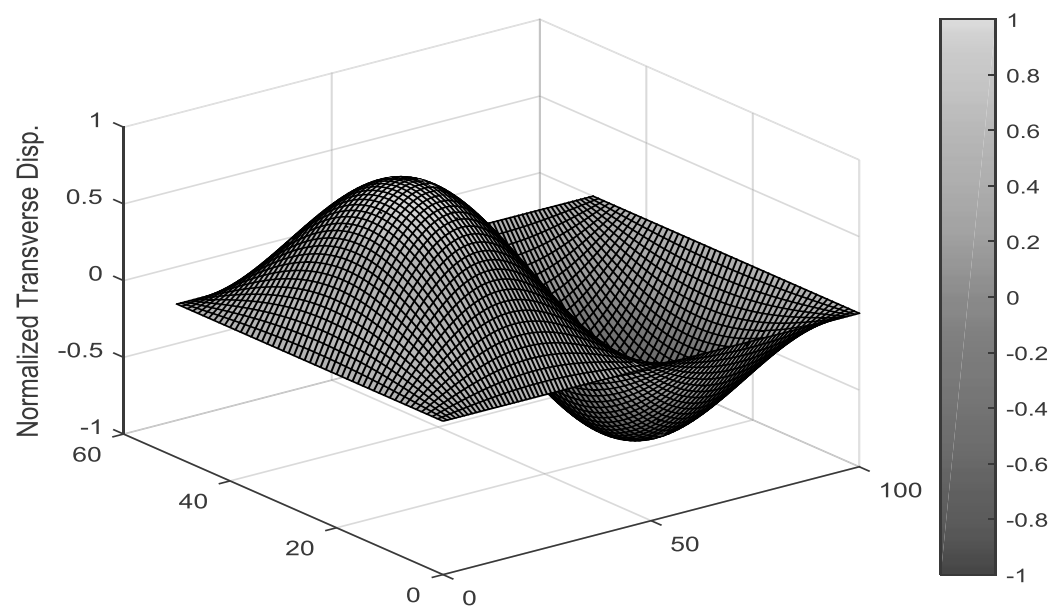

Figure 3. Thermal Buckling mode for symmetric cross-ply square plate, mode $(M=2, N=1)$, No. of layers $=8, a / h=4, a / b=2$.

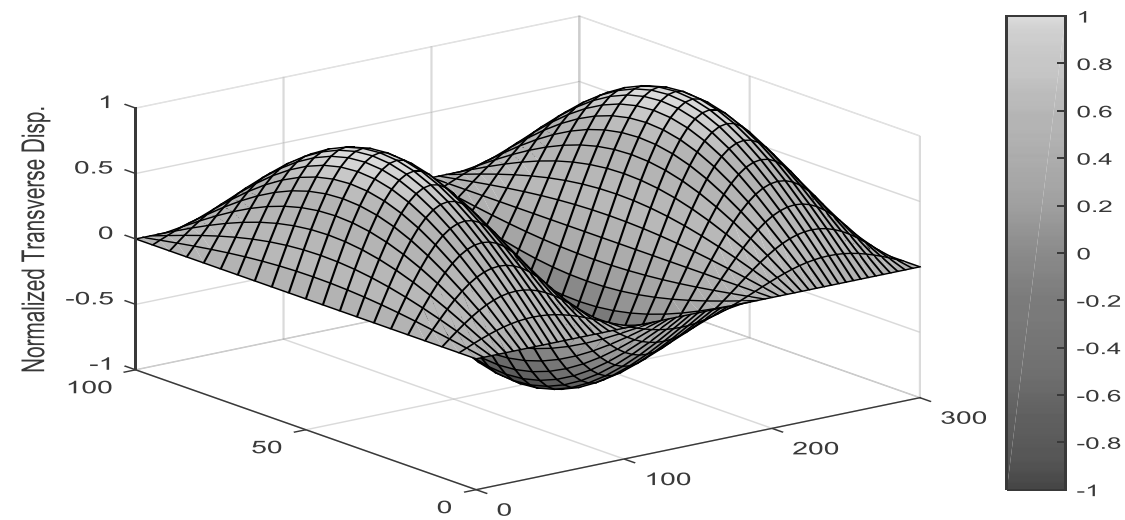

Figure 4. Thermal Buckling mode for symmetric cross-ply square plate, mode $(M=3, N=1)$, No. of layers $=8, a / h=4, a / b=3$.

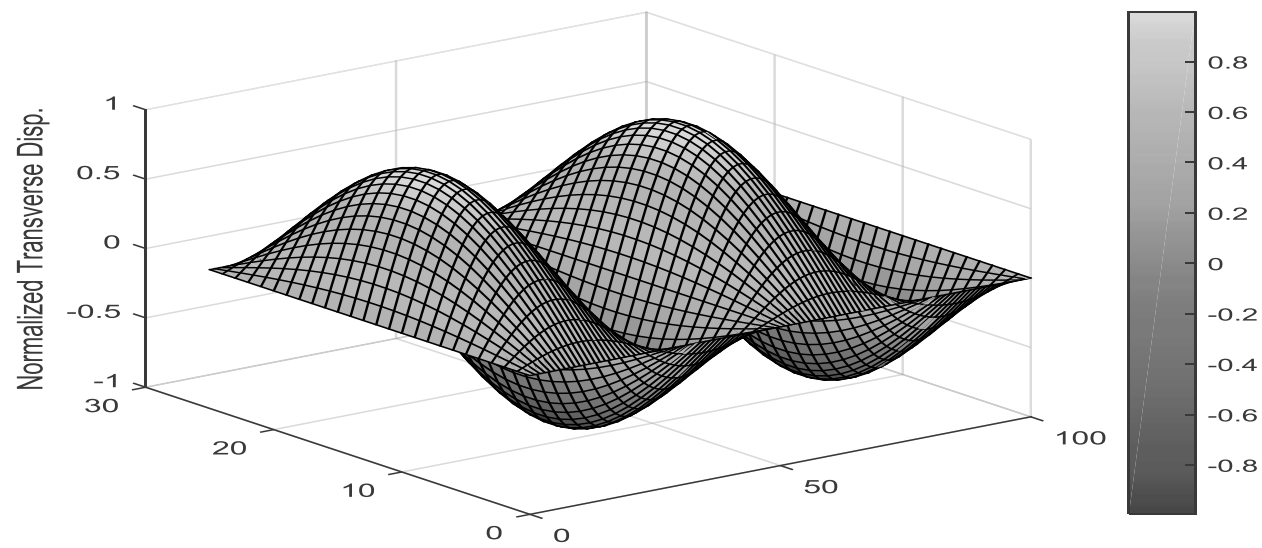

Figure 5. Thermal Buckling mode for symmetric cross-ply square plate, mode $(M=4, N=1)$, No. of layers $=8, \mathrm{a} / \mathrm{h}=4, \mathrm{a} / \mathrm{b}=4$. 
Tables 5, 6, and 7 show effect of changing $\left(\mathrm{E}_{1} / \mathrm{E}_{2}\right)$ on critical temperature for 4,8 , and 20 layers symmetric and antisymmetric cross-ply plates, since stiffness increase when increasing orthotropic ratio therefore normalized critical temperature decrease $\left(\mathrm{D}_{22}\right.$ increase). Material used for all present work results are as follows: $\mathrm{E}_{1} / \mathrm{E}_{2}=25, \mathrm{G}_{12}=\mathrm{G}_{13}=.5 \mathrm{E}_{2}$, $\mathrm{G}_{23}=.2 \mathrm{E}_{2}, v_{12}=0.25, \alpha_{2} / \alpha_{1}=3$, except when mentioned.

Table 5. Effect of $\left(E_{1} / E_{2}\right)$ on normalized critical temperature $\left(T^{*} c r=T^{*} a^{2 *} h / \pi^{2 *} D_{22}\right)$ for symmetric cross-ply square plate, mode $(\mathrm{M}=1, \mathrm{~N}=1)$, No. of layers $=4$.

\begin{tabular}{|c|c|c|c|c|c|c|}
\hline \multirow{2}{*}{$\mathbf{a} / \mathbf{H}$} & \multicolumn{3}{|c|}{ Symmetric layers } & \multicolumn{3}{c|}{ Antisymmetric layers } \\
\cline { 2 - 7 } & \multicolumn{3}{|c|}{$\mathbf{E}_{\mathbf{1}} / \mathbf{E}_{\mathbf{2}}$} & \multicolumn{3}{c|}{$\mathbf{E}_{\mathbf{2}}$} \\
\cline { 2 - 7 } & 10 & 25 & 40 & 10 & 25 & 40 \\
\hline 4 & 0.16029 & 0.0533 & 0.02704 & 0.07105 & 0.01974 & 0.009339 \\
\hline 10 & 0.32783 & 0.14199 & 0.08099 & 0.12715 & 0.04642 & 0.02591 \\
\hline 100 & 0.42475 & 0.2364 & 0.16295 & 0.14983 & 0.06298 & 0.03974 \\
\hline
\end{tabular}

Table 6. Effect of $\left(E_{1} / E_{2}\right)$ on normalized critical temperature $\left(T^{*} \mathrm{cr}=T^{*} \mathrm{a}^{2 *} \mathrm{~h} / \pi^{2 *} \mathrm{D}_{22}\right)$ for symmetric cross-ply square plate, mode $(\mathrm{M}=1, \mathrm{~N}=1)$, No. of layers $=8$.

\begin{tabular}{|c|c|c|c|c|c|c|}
\hline \multirow{2}{*}{$\mathbf{a} / \mathbf{H}$} & \multicolumn{3}{|c|}{ Symmetric layers } & \multicolumn{3}{c|}{ Antisymmetric layers } \\
\cline { 2 - 7 } & \multicolumn{3}{|c|}{$\mathbf{E}_{\mathbf{1}} / \mathbf{E}_{\mathbf{2}}$} & \multicolumn{3}{c|}{$\mathbf{E}_{\mathbf{1}} / \mathbf{E}_{\mathbf{2}}$} \\
\cline { 2 - 7 } & 10 & 25 & 40 & 10 & 25 & 40 \\
\hline 4 & 0.10535 & 0.03113 & 0.01503 & 0.07491 & 0.021072 & 0.010034 \\
\hline 10 & 0.19832 & 0.07787 & 0.04386 & 0.13706 & 0.051216 & 0.02864 \\
\hline 20 & 0.22842 & 0.101379 & 0.062813 & 0.155864 & 0.064974 & 0.039749 \\
\hline 100 & 0.24022 & 0.11255 & 0.07337 & 0.16304 & 0.071124 & 0.043543 \\
\hline
\end{tabular}

Table 7. Effect of $\left(E_{1} / E_{2}\right)$ on normalized critical temperature $\left(T^{*} c r=T^{*} a^{2 *} h / \pi^{2 *} D_{22}\right)$ for symmetric cross-ply square plate, mode $(\mathrm{M}=1, \mathrm{~N}=1)$, No. of layers $=20$.

\begin{tabular}{|c|c|c|c|c|c|c|}
\hline \multirow{2}{*}{$\mathbf{a} / \mathbf{H}$} & \multicolumn{3}{|c|}{ Symmetric layers } & \multicolumn{3}{c|}{ Antisymmetric layers } \\
\cline { 2 - 7 } & \multicolumn{3}{|c|}{$\mathbf{E}_{\mathbf{1}} / \mathbf{E}_{\mathbf{2}}$} & \multicolumn{3}{c|}{$\mathbf{E}_{\mathbf{1}} / \mathbf{E}_{\mathbf{2}}$} \\
\cline { 2 - 7 } & 10 & 25 & 40 & 10 & 25 & 40 \\
\hline 4 & 0.08628 & 0.02478 & 0.01187 & 0.07604 & 0.02146 & 0.010239 \\
\hline 10 & 0.159375 & 0.06094 & 0.03422 & 0.13984 & 0.05255 & 0.029412 \\
\hline 20 & 0.181889 & 0.077915 & 0.04797 & 0.15929 & 0.066935 & 0.041027 \\
\hline 100 & 0.190541 & 0.0856 & 0.05517 & 0.16673 & 0.07340 & 0.047027 \\
\hline
\end{tabular}

\section{CONCLUSIONS}

Thermal buckling solution of thick and thin cross-ply composite plates is developed using new displacement function suggested by Mantari et al. for static and free vibration analyses of composite plate (they used $\mathrm{m}=0.5$ ), but " $\mathrm{m}$ " is changed in present work to ' $\mathrm{m}=.05$ ', at which it gives results that agree well with those of the $3 \mathrm{D}$ elasticity theory with maximum discrepancy (5.05\%) and other plate shear theories. As expected critical temperature is decreased 
as thickness ratio, thermal coefficient ratio $\alpha_{2} / \alpha_{1}$ and aspect ratio increased, while the buckling temperature increases when $E_{1} / E_{2}$ increases and is larger for thick than thin plates, and also antisymmetric cross-ply will buckle with a temperature larger than those for symmetric ply.

Mode number of thermal buckling for simply supported cross-ply plate does not change when using this new displacement field.

\section{REFERENCES}

A. Menasria, A. Bouhadra, Abdelouahed Tounsi, Abdelmoumen Anis Bousahla and Samy R. Mahmoud. (2017). A new and simple HSDT for thermal stability analysis of FG sandwich plates. Steel and Composite Structures, 25: 157-175.

A. Bouhadra, Abdelouahed Tounsi, Abdelmoumen Anis Bousahla \& Samir Benyoucef and Samy R. Mahmoud. (2018). Improved HSDT accounting for effect of thickness stretching in advanced composite plates. Structural Engineering and Mechanics, 66: 61-73.

A. Younsi, Abdelouahed Tounsi, Zaoui Fatima Zohra, Abdelmoumen Anis Bousahla and Samy R. Mahmoud. (2018). Novel quasi-3D and 2D shear deformation theories for bending and free vibration analysis of FGM plates. Geomechanics and Engineering, 14: 519-532.

Abdelbaki Chikh, Abdelouahed Tounsi, Habib Hebali and S.R. Mahmoud. (2017). Thermal buckling analysis of cross-ply laminated plates using a simplified HSDT. Smart Structures and Systems, 19(3): 289-297.

Abdelkader Mahmoudi, Samir Benyoucef, Abdelouahed Tounsi, Abdelkader Benachour, El Abbas Adda Bedia, SR Mahmoud. ( 2019). A refined quasi-3D shear deformation theory for thermo-mechanical behavior of functionally graded sandwich plates on elastic foundations. Journal Of Sandwich Structures \& Materials, 21(6): 1906-1929.

Adnan Naji Jameel, Ibtehal Abbas Sadiq and Hasanain Ibraheem Nsaif. 2012. Buckling analysis of composite plates under thermal and mechanical loading, Journal of Engineering, 12(18):1365-1390.

Ahmed K. Noor and W. Scott Burton. 1992. Three-dimensional solutions for the thermal buckling and sensitivity derivatives of temperature-sensitive muitilayered angle-ply plates. Journal of Applied Mechanics (Transactions of the ASME), 59: 848-856.

Ahmed K. Noor and W. Scott Burton 1992. Three-dimensional solutions for thermal buckling of multilayered anisotropic plates. Journal of Engineering Mechanics, 118(4): 683-701.

Ahmed K. Noor and Jeanne M. Peters 1992. Thermomechanical buckling of multilayered composite plates. Journal of Engineering Mechanics, 118: 351-366.

Ait Atmane Hassen, Abdelouahed Tounsi and Fabrice Bernard. (2017). Effect of thickness stretching and porosity on mechanical response of a functionally graded beams resting on elastic foundations. International Journal of Mechanics and Materials in Design, 13: 71-84.

Amina Attia, Abdelmoumen Anis Bousahla, Abdelouahed Tounsi, Samy R. Mahmoud and Afaf S. Alwabli. (2018). A refined four variable plate theory for thermoelastic analysis of FGM plates resting on variable elastic foundations. Structural Engineering and Mechanics, 65: 453-464.

Bellifa Hichem, Ahmed Bakora, Abdelouahed Tounsi, Abdelmoumen Anis Bousahla and Samy R. Mahmoud. (2017). An efficient and simple four variable refined plate theory for buckling analysis of functionally graded plates. Steel and Composite Structures, 25: 257-270.

Behrouz Karami, Maziar Janghorban, Davood Shahsavari and Abdelouahed Tounsi. (2018). A size-dependent quasi-3D model for wave dispersion analysis of FG nanoplates. Steel and Composite Structures. 28: 99-110.

Boulefrakh Laid, Hebali Habib, Chikh Abdelbaki, Abdelmoumen Anis Bousahla, Abdelouahed Tounsi and Samy R. Mahmoud. (2019). The effect of parameters of visco-Pasternak foundation on the bending and vibration properties of a thick FG plate. Geomechanics and Engineering, 18: 161-178.

Djaloul Zarga, Abdelouahed Tounsi, Abdelmoumen Anis Bousahla, Fouad Bourada and S.R. Mahmoud. (2019). Thermomechanical bending study for functionally graded sandwich plates using a simple quasi-3D shear deformation theory. Steel and Composite Structures, 32(3): 389-410.

Emad Q. Hussein and Sara J. Alasadi. 2018. Experimental and theoretical stress analysis investigation for composite plate under 
thermal load. Kufa Journal of Engineering, 9(1): 205-221.

F. El-Haina, Ahmed Bakora, Abdelmoumen Anis Bousahla, Abdelouahed Tounsi and Samy R. Mahmoud. (2017). A simple analytical approach for thermal buckling of thick functionally graded sandwich plates. Structural Engineering and Mechanics, 63: $585-595$.

Fouad Bourada, K. Amara, Abdelmoumen Anis Bousahla, Abdelouahed Tounsi, Samy R. Mahmoud. (2018). A novel refined plate theory for stability analysis of hybrid and symmetric S-FGM plates. Structural Engineering and Mechanics, 68: 661-675.

Fouad Bourada, Abdelmoumen Anis Bousahla, Mohamed Bourada, Abdelghani Azzaz, Amina Zinata and Abdelouahed Tounsi. (2019). Dynamic investigation of porous functionally graded beam using a sinusoidal shear deformation theory. Wind and Structures, 28(1): 019-30.

H. Fourn, Ait Atmane Hassen, Mohamed Bourada, Abdelmoumen Anis Bousahla, Abdelouahed Tounsi, and Samy R. Mahmoud. (2018). A novel four variable refined plate theory for wave propagation in functionally graded material plates. Steel and Composite Structures, 27: 109-122.

H.H.Abdelaziz, M. Ait Amar Meziane, Abdelmoumen Anis Bousahla, Abdelouahed Tounsi, Samy R. Mahmoud and Afaf S. Alwabli. (2017). An efficient hyperbolic shear deformation theory for bending, buckling and free vibration of FGM sand wich plates with various boundary conditions. Steel and Composite Structures, 25: 693-704.

Hadjira Hellal, Mohamed Bourada, Hebali Habib, Fouad Bourada, Abdelouahed Tounsi, Abdelmoumen Anis Bousahla, Samy R. Mahmoud. (2019). Dynamic and stability analysis of functionally graded material sandwich plates in hygrothermal environment using a simple higher shear deformation theory. Journal of Sandwich Structures \& Materials. doi. org/10.1177/1099636219845841.

Heinz W. Bargmann 1985. Thermal buckling of elastic plates. Journal of Thermal Stresses, 8(1): 71-98.

Houdayfa Ounis and Mohamed-Ouejdi Belarbi 2017. On the thermal buckling behaviour of laminated composite plates with cut-outs. Journal Of Applied Engineering Science \& Technology, 3(2): 63-69.

J.L. Mantari, A.S. Oktem and C. Guedes Soares. 2011. Static and dynamic analysis of laminated composite and sandwich plates and shells by using a new higher-order shear deformation theory. Composite Structures, 94: 37-49.

J.N. Reddy 2004. Mechanics of laminated composite plates and shells. second edition CRC press.

L.X. SUN and T.R. HSIJ 1990. Thermal buckling of laminated composite plates with transverse shear deformation. Computers and Structures, 36(5): 883-889.

Le-Chung Shiau, Shih-Yao Kuo and Cheng-Yuan Chen 2010. Thermal buckling behavior of composite laminated plates. Composite Structures, 92: 508-514.

Lynda Amel Chaabane, Fouad Bourada, Mohamed Sekkal, Sara Zerouati, Fatima Zohra Zaoui, Abdeldjebbar Tounsi, Abdelhak Derras, Abdelmoumen Anis Bousahla and Abdelouahed Tounsi. (2019). Analytical study of bending and free vibration responses of functionally graded beams resting on elastic foundation. Structural Engineering and Mechanics, 71(2): 185-196.

M. Benchohra, H. Driz, Ahmed Bakora, Abdelouahed Tounsi, E.A. Bedia and Samy R. Mahmoud. (2018). A new quasi-3D sinusoidal shear deformation theory for functionally graded plates. Structural Engineering and Mechanics, 65: 19-31.

M. Cetkovic. 2016. Thermal buckling of laminated composite plates using layerwise displacement model. Composite Structures, 142: $238-253$.

M. Mansour Mohieddin Ghomshei and Amin Mahmoudi 2010. Thermal buckling analysis of cross-ply laminated rectangular plates under nonuniform temperature distribution: A differential quadrature approach, Journal of Mechanical Science and Technology, 24(12): 2519-2527.

Marina Ćetković and Lénárt György 2016. Thermo-elastic stability of angle-ply laminates application of layerwise finite elements. Structural Integrity and Life, 16(1): 43-48.

Mohammed Medani, Abdelillah Benahmed, Mohamed Zidour, Houari Heireche, Abdelouahed Tounsi, Abdelmoumen Anis Bousahla, Abdeldjebbar Tounsi and S.R. Mahmoud. (2019). Static and dynamic behavior of (FG-CNT) reinforced porous sandwich plate using energy principle. Steel and Composite Structures, 32(5): 595-610.

Mohamed Bourada, Abdelouahed Tounsi, Mohammed Sid Ahmed Houari and El Abbes Adda Bedia 2011. A new four- 
variable refined plate theory for thermal buckling analysis of functionally graded sandwich plates. Journal of Sandwich Structures and Materials, 14(1): 5-33.

Moussa Abualnour, Mohammed Sid Ahmed Houari, Abdelouahed Tounsi, El Abbes Adda Bedia and Samy R. Mahmoud. (2018). A novel quasi-3D trigonometric plate theory for free vibration analysis of advanced composite plates. Composite Structures, 184: 688-697.

R. Vescovini, M. D'Ottavio, L. Dozio and O. Polit. 2017. Thermal buckling response of laminated and sandwich plates using refined 2-D models. Composite Structures, 176(15): 313-328.

Rafik Meksi, Samir Benyoucef, Abdelkader Mahmoudi, Abdelouahed Tounsi, El Abbas Adda Bedia and SR Mahmoud. (2019). An analytical solution for bending, buckling and vibration responses of FGM sandwich plates. Journal Of Sandwich Structures \& Materials, 21(2): 727-757.

Rajiv Kumar, Amit Sharma and Rajesh Kumar. 2013. Thermal buckling analysis of a laminated composite plate resting on elastic foundation using stochastic finite element method based on micromechanical model. International Journal of Engineering, Business and Enterprise Applications, 1: 133-139.

Riad Hamza-Cherif, Mustapha Meradjah, Mohamed Zidour, Abdelouahed Tounsi \& Samir Belmahi, and Bensattalah Tayeb. (2018). Vibration Analysis of Nano Beam Using Differential Transform Method Including Thermal Effect. Journal of Nano Research, 54: 1-14.

Sabrina Boutaleb, Kouider Halim Benrahou, Ahmed Bakora, Ali Algarni, Abdelmoumen Anis Bousahla, Abdelouahed Tounsi, Abdeldjebbar Tounsi and S.R. Mahmoud. (2019). Dynamic analysis of nanosize FG rectangular plates based on simple nonlocal quasi 3D HSDT. Advances in Nano Research, 7(3): 189-206.

Shu Xiaoping and Sun Liangxin. 1994. Thermo mechanical buckling of laminated composite plates with higher-order transverse shear deformation. Computers \& Structures, 53: 1-7.

Wael R. Abdul-Majeed, Muhsin J. Jweeg and Adnan N. Jameel. 2011. Thermal buckling of rectangular plates with different temperature distribution using strain energy method. Journal of Engineering, 5(17): 1047-1065.

Youcef Beldjelili, Abdelouahed Tounsi and Samy Mahmoud. 2016. Hygro-thermo-mechanical bending of S-FGM plates resting on variable elastic foundations using a four-variable trigonometric plate theory. Smart Structures and Systems, 18: 755-786.

Yufeng Xing and Zekun Wang. 2017. Closed form solutions for thermal buckling of functionally graded rectangular thin plates. Applied Sciences, 7: 1256.

Zaoui Fatima Zohra, Djamel Ouinas and Abdelouahed Tounsi. (2019). New 2D and quasi-3D shear deformation theories for free vibration of functionally graded plates on elastic foundations. Composites Part B Engineering, 159: 231-247.

Zoulikha Boukhlif, Mohammed Bouremana, Fouad Bourada, Abdelmoumen Anis Bousahla, Mohamed Bourada, Abdelouahed Tounsi and Mohammed A. Al-Osta. (2019). A simple quasi-3D HSDT for the dynamics analysis of FG thick plate on elastic foundation. Steel and Composite Structures, 31( 5): 503-516. 